\title{
PEPra: pour la prévention au cabinet de premier recours
}

\author{
Barbara Zosso ${ }^{a}$, Carlos B. Quinto ${ }^{b}$, Stefan Neuner-Jehle ${ }^{c}$, Barbara Weild \\ ${ }^{a}$ Collaboratrice scientifique/responsable de projet division santé publique/ professions de la santé; b Dr méd., membre du Comité central de la FMH, \\ responsable du département Santé publique/professions de la santé; ${ }^{c}$ Prof. Dr méd., responsable Chronic Care, Institut de médecine de famille, Université et \\ Hôpital universitaire de Zurich; ' Cheffe de la division Santé publique/professions de la santé, FMH
}

\section{Le projet PEPra - La prévention au cabinet médical fondée sur les preuves porté par la FMH et d'autres partenaires et soutenu par la Fondation Promotion Santé Suisse vise à établir jusqu'en 2024 un ensemble de mesures coordonnées pour la préven- tion au cabinet médical.}

"La prévention est un thème important de la prise en charge dans les cabinets médicaux de premier recours. Une consultation sur deux porte sur l'obésité, le diabète, les maladies respiratoires, l'addiction, etc. Des sujets difficiles à aborder et souvent source de frustrations.» C'est ce que déclarent de nombreux médecins de premier recours quand ils s'expriment sur le thème de la prévention au cabinet médical [1]. Plusieurs raisons expliquent pourquoi ces sujets sont ressentis comme difficiles à aborder ou sources de frustrations: premièrement, les mesures préventives visent souvent à réduire les facteurs de risque et à renforcer les facteurs de protection, ce qui ne peut généralement être obtenu que par des changements de comportement, pour la plupart difficiles à mettre en œuvre car indissociablement liés à la motivation du patient mais aussi à des revers et à des échecs [2]. Cela demande donc beaucoup de patience de la part de chacun. Deuxièmement, le conseil en matière de prévention exige du temps, qui a été limité dans le cadre de la consultation médicale par les interventions tarifaires du Conseil fédéral. En troisième lieu, les outils spécifiques qui existent pour la consultation [3] sont souvent inconnus ou difficiles à trouver; tout comme les offres en amont et en aval dans le domaine de la prévention, p. ex. promotion de l'activité physique, conseil à l'arrêt du tabagisme, conseil en matière d'addiction, etc.

Pourtant, malgré ces défis, la prévention au cabinet médical présente aussi beaucoup de potentiel: pour 75 à $80 \%$ de la population suisse qui se rend au moins une fois par an chez le médecin, c'est la possibilité d'être sensibilisée à la prévention. Les médecins peuvent motiver leurs patients à changer de comportement ou à adopter d'autres mesures préventives, les conseiller et les épauler, et ainsi contribuer à réduire les maladies évitables. La prévention fait partie de l'activité médicale [4]. Elle ne peut et ne doit pas être entièrement déléguée à d'autres acteurs, d'autant plus que la crédibilité des médecins et le cadre du cabinet médical offrent des conditions optimales pour le travail de prévention. C'est pourquoi la FMH s'engage dans le projet PEPra (La prévention au cabinet médical fondée sur les preuves).

\section{Que faut-il donc faire?}

Pour que les médecins de premier recours et leurs assistantes médicales (AM) ou coordinatrices en médecine ambulatoire (CMA) puissent mettre en œuvre la prévention au cabinet médical, il faut s'occuper des difficultés mentionnées au début. Les conditions cadre structurelles et financières pour rendre possible et encourager la prévention au cabinet médical doivent être créées. L'amélioration de ces conditions cadre est une préoccupation majeure des sociétés de discipline et associations de médecins à laquelle on n'accorde pas (encore) suffisamment d'attention au niveau politique. Par ailleurs, les médecins et les assistantes médicales doivent disposer des «instruments» appropriés. C'est ce à quoi s'attelle le projet PEPra: créer un ensemble de mesures coordonnées pour la prévention au cabinet médical en collaboration avec les médecins et les assistantes médicales.

\section{Que propose PEPra?}

PEPra ne développe pas une nouvelle approche, mais s'appuie sur des programmes et approches nationaux déjà développés par le corps médical pour la prévention au cabinet médical, notamment Coaching santé du CMPR, Vivre sans tabac, le programme Paprica pro- 


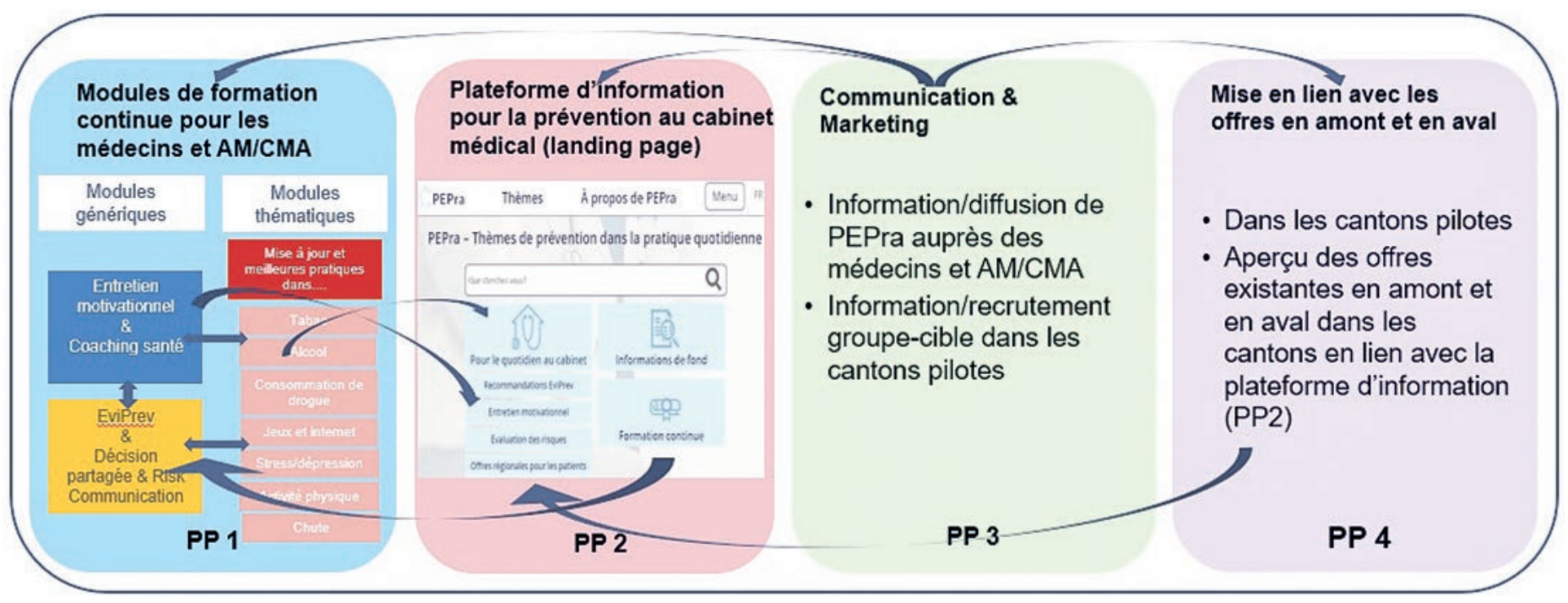

Figure 1: L'ensemble de mesures PEPra avec ses quatre produits/projets partiels. Les flèches symbolisent l'interdépendance des différents domaines et produits (C Division Santé publique de la FMH).

posé par Unisanté, les deux programmes plus anciens développés par la FMH (Crise et suicide et Intervention brève lors de consommation d'alcool à problème) ainsi que les Recommandations EviPrev de l'association du même nom. Pour cela, PEPra s'attache à réunir entre eux, mais de manière perméable, les axes thématiques sur la base desquels sont conçus un grand nombre de ces programmes et implique leurs organes responsables dans le développement. Les résultats des évaluations des programmes réalisées jusqu'ici sont pris en compte tout comme les résultats d'autres sondages et études relatifs aux besoins du corps médical en matière de prévention au cabinet médical $[2,5]$.

L'ensemble de mesures coordonnées se décline en quatre produits (fig. 1).

PEPra repose sur des programmes et approches éprouvés et développés par le corps médical pour la prévention au cabinet médical.

Les cours modulaires (projet partiel 1) pour les médecins de premier recours et les assistantes médicales constituent l'élément central. L'entretien motivationnel (EM) avec son approche ciblée visant à susciter les motivations intrinsèques du patient y joue un rôle essentiel [6] et peut être considéré comme un élément générique. L'efficacité de l'entretien motivationnel a été analysée dans plus de 200 études randomisées contrôlées. Il s'est avéré que cette approche est plus efficace que les interventions standards, même de courte durée (<10 min) [7]. Le projet PEPra a pour but de développer l'entretien motivationnel en association avec l'approche Coaching santé du CMPR et d'en faire un module de formation continue générique pour la prévention au cabinet médical. Les recommandations EviPrev sont un autre élément générique qui montrent, sur la base des preuves scientifiques disponibles, chez quel patient il convient d'aborder quel thème de prévention et à quel moment. Ces recommandations font partie d'un autre module générique incluant d'autres techniques de communication et de conseil telles que la décision partagée et la communication des bénéfices et risques. L'offre de formation continue est complétée par des modules thématiques [8] portant sur des méthodes d'intervention et de conseil éprouvées et des outils concrets sur les thèmes respectifs. Lors des formations continues, le "quoi» (théorie, connaissances scientifiques) est systématiquement associé au «comment» (méthodes de communication et de conseil, attitudes, outils, etc.). De plus, une large place est accordée à la mise en situation. L'offre vise donc à approfondir les compétences dans les domaines qui sont souvent considérés comme difficiles: aborder, motiver, soutenir et accompagner les patients. Les modules seront testés dès 2022 dans quatre cantons sous forme de projets pilotes.

En complément, une plateforme d'information (landing page) couvrant une multitude de sujets sera mise sur pied pour la prévention au cabinet médical. Elle offrira un accès aux utilisateurs de PEPra et comprendra dans un premier temps les thèmes des programmes sur lesquels se fonde PEPra. Elle sera cependant conçue de façon à pouvoir intégrer progressivement d'autres thèmes. Elle comportera en particulier des outils, guides et recommandations facilement accessibles qui pourront être utilisés au cabinet médical dans le 


\begin{tabular}{ll}
\hline Tableau 1: Fiche descriptive du projet «PEPra - La prévention au cabinet médical fondée sur les preuves». \\
\hline Contexte & $\begin{array}{l}\text { Stratégie nationale Prévention des maladies non transmissibles (stratégie MNT), groupe de mesures } \\
2 \text { «Prévention dans le domaine des soins (PDS)» }\end{array}$ \\
\hline Promotion du projet & $\begin{array}{l}\text { Fondation Promotion Santé Suisse (FPSS), Fonds «Prévention dans le domaine des soins» (PDS), } \\
\text { dans le cadre d'une «collaboration directe FPSS/OFSP-FMH» (en complément au soutien proactif du } \\
\text { projet) }\end{array}$ \\
\hline Promoteurs du projet & FMH \\
\hline Partenaires & $\begin{array}{l}\text { Collège de médecine de premier recours CMPR, Unisanté, Praticien Addiction Suisse, Association } \\
\text { EviPrev, Conférence des délégués cantonaux aux problèmes des addictions CDCA, Association } \\
\text { suisse alémanique des assistantes médicales SVA/Association romande des assistantes médicales } \\
\text { aram }\end{array}$ \\
\hline Durée du projet & 2020 à 2024 \\
\hline Financement FPSS & CHF 1,725 million (y compris l'évaluation) \\
\hline Budget global & env. CHF 2,2 millions (sans les ressources internes à la FMH en dehors de la direction du projet) \\
\hline Evaluation & Interface \& IHAM\&CC Lucerne \\
\hline Cantons pilotes & Bâle-Ville, Fribourg, Saint-Gall, Lucerne \\
\hline
\end{tabular}

cadre de l'entretien direct avec les patients. De plus, elle servira aussi de plateforme d'inscription pour les formations continues PEPra. La réalisation est prévue en 2021.

Du fait que la durée des consultations est limitée et que les changements de comportement nécessitent du temps, il peut s'avérer judicieux de motiver les patients à solliciter des offres existantes dans le domaine de la prévention au sein ou en dehors du domaine des soins (conseil à l'arrêt du tabagisme, promotion de l'activité

\section{Une plateforme d'information couvrant une multitude de sujets sera mise sur pied pour la prévention au cabinet médical.}

physique, conseil en matière d'addiction, etc.). Les cantons pilotes établiront dans le cadre du projet partiel 4 des aperçus des offres existantes en amont et en aval dans leur région (ou développeront les aperçus existant partiellement) et les mettront si possible à disposition sur la plateforme d'information.

\section{Conclusion}

La prévention au cabinet médical reste un défi. L'ensemble de mesures qui sera établi dans le cadre du projet vise à soutenir les médecins et les assistantes médicales dans ce travail. L'évaluation du projet montrera dans quelle mesure il sera possible de satisfaire à cette exigence. La durabilité dépendra essentiellement de la capacité d'améliorer parallèlement les conditions pour la prévention, c'est-à-dire les conditions cadre structurelles et financières. La FMH poursuivra donc ses efforts au niveau politique dans ce domaine.

\section{Références}

1 Prévention sert de terme générique pour la prévention primaire, secondaire, tertiaire et quaternaire.

2 Zyska Cherix A. Quelle perception ont les médecins de famille en Suisse de leur rôle dans la prévention et la promotion de la santé? Université de Lausanne 2014. http://serval.unil.ch

3 P. ex. les outils pour le coaching santé (https://www.gesundheitscoaching-khm.ch/fr), les recommandations EviPrev (https:// eviprev.ch/), le manuel Praticien Addiction Suisse (https://www.praxis-suchtmedizin.ch/) et bien d'autres.

4 LPMéd, art. 4 al.1, art. 6. al. 1 let. a, art. 8 let. h; PROFILES, https:// www.profilesmed.ch/ (accès le 10.11.2020).

5 Zufferey-Caiata M, De Pietro C. Evaluation du processus de mise en œuvre du projet-pilote Girasole. Rapport final à l'attention de l'Office fédéral de la santé publique. SUPSI, Lugano/Manno 2018.

6 Oetterli M, Hanimann A, Balthasar A. Evaluation des Pilotprojekts im Kanton Tessin: Synthesebericht. Interface, Luzern 2019.

7 Neuner-Jehle S. Schlussbericht über das vom BAG subventionierte Projekt «Gesundheitscoaching KHM» 2015-2018. 2018.

8 Oetterli M, Hanimann A, Büchler C. Zielgruppenbefragung zum Programm Gesundheitscoaching KHM. Bericht zuhanden des Stiftungsrats des Kollegiums für Hausarztmedizin. Interface, Luzern 2019.

9 Association EviPrev, «FocusPrev I» et «FocusPrev II», 2006-2009, https://eviprev.ch/downloads/

10 Steiger D, Brauchbar M. Analyse IST-Zustand präventiver Angebote im Sinne der Prävention in der Gesundheitsversorgung (PGV) in der ambulanten Praxis, EvalueScience, im Auftrag des BAG. 2018.

11 Miller WR, Rollnick R. Motivational Interviewing. Preparing People for Change. 2002

12 Gache P, Sommer J. Rev Med Suisse. 2018;591(14):229-30.

13 Les thèmes de ces modules se limitent pour le moment aux thèmes des programmes et approches sur lesquels se fonde PEPra ainsi que sur d'autres thèmes de l'addiction: tabac, alcool, consommation de drogue, jeux et internet, stress/dépression, activité physique, prévention des chutes.
FMH

Division Santé publique/ professions de la santé Nussbaumstrasse 29 Case postale

CH-3000 Berne 15 public.health[at]fmh.ch

\section{Remarque}

Cet article paraît en même temps dans le numéro 12 de la revue Primary and Hospital Care https://primary-hospital-care.ch/fr/article/doi/phc-f.2020.10331. 